\title{
Stereotactic Body Radiation Therapy for Oligometastatic Prostate Cancer
}

\author{
Jonathan L. Muldermans, BS ${ }^{\star}$, Lindsay B. Romak, MD $^{\dagger}$, Eugene D. Kwon, MD ${ }^{\ddagger}, \S$, Sean S. \\ Park, MD, $\mathrm{PhD}^{\dagger}$, and Kenneth R. Olivier, $\mathrm{MD}^{\dagger}$
}

${ }^{*}$ F. Edward Hébert School of Medicine, Uniformed Services University of the Health Sciences, Bethesda, Maryland tDepartment of Radiation Oncology, Mayo Clinic, Rochester, Minnesota ‡Department of Urology, Mayo Clinic, Rochester, Minnesota §Department of Immunology, Mayo Clinic, Rochester, Minnesota

\begin{abstract}
Purpose-To review outcomes of patients with oligometastatic prostate cancer (PCa) treated with stereotactic body radiation therapy (SBRT) and to identify variables associated with local failure.
\end{abstract}

Methods and Materials-We retrospectively reviewed records of patients treated with SBRT for oligometastatic PCa. Metastasis control (ie, control of the treated lesion, MC), biochemical progression-free survival, distant progression-free survival, and overall survival were estimated with the Kaplan-Meier method.

Results-Sixty-six men with 81 metastatic PCa lesions, 50 of which were castrate-resistant, were included in the analysis. Lesions were in bone $(n=74)$, lymph nodes $(n=6)$, or liver $(n=1)$. Stereotactic body radiation therapy was delivered in 1 fraction to 71 lesions (88\%), at a median dose of $16 \mathrm{~Gy}$ (range, 16-24 Gy). The remaining lesions received $30 \mathrm{~Gy}$ in 3 fractions ( $\mathrm{n}=6$ ) or 50 Gy in 5 fractions $(n=4)$. Median follow-up was 16 months (range, 3-49 months). Estimated MC at 2 years was $82 \%$. Biochemical progression-free survival, distant progression-free survival, and overall survival were $54 \%, 45 \%$, and $83 \%$, respectively. On multivariate analysis, only the dose of SBRT was significantly associated with MC; lesions treated with 16 Gy had $58 \% \mathrm{MC}$, and those treated with $\geq 18$ Gy had $95 \% \mathrm{MC}$ at 2 years $(P \leq 001)$. At 2 years, MC for lesions treated with $\geq 18$ Gy $(n=21)$ was $88 \%$. No patient treated with $\geq 18$ Gy in a single fraction or with any multifraction regimen had local failure. Six patients (9\%) had grade 1 pain flare, and $2(3 \%)$ had grade 2 pain flare. No grade 2 or greater late toxicities were reported.

Conclusions-Stereotactic body radiation therapy for patients with oligometastatic prostate cancer provided optimal metastasis control and acceptable toxicity with doses $\geq 18 \mathrm{~Gy}$. Biochemical progression-free survival was $54 \%$ at 16 months with the inclusion of SBRT in the

Reprint requests to: Kenneth R. Olivier, MD, Department of Radiation Oncology, Mayo Clinic, 200 First St SW, Rochester, MN 55905. Tel: (507) 284-4561; olivier.kenneth@ mayo.edu.

J.L.M. and L.B.R. contributed equally to this work.

S.S.P. and K.R.O. contributed equally to this work.

Conflict of interest: none. 
treatment regimen. Stereotactic body radiation therapy should be considered in patients with castration-refractory, oligometastatic prostate cancer who have limited options for systemic therapy.

\section{Introduction}

Prostate cancer ( $\mathrm{PCa})$ is the second leading cause of cancer-related death and is the most common noncutaneous cancer in men, with approximately 1 in 6 men ultimately diagnosed with the disease (1). For men with metastatic PCa, systemic therapy, including androgen deprivation therapy and chemotherapy, is considered the standard of care. The systemic options for patients with castration-refractory metastatic PCa have recently improved, with the addition of agents such as abiraterone (2) and enzalutamide (3), which result in modest improvement in duration of prostate-specific antigen (PSA) control. However, not all patients or all metastases respond to these drugs. Metastasis-directed therapies, such as radiation, ablation, or surgery, for such patients and lesions may be used.

Decisions regarding treatment of metastatic lesions were traditionally directed by present or impending patient symptoms. More recently, interest has increased in the potential role of definitive metastasis-directed therapy for men with oligometastatic disease, defined as few (often 25 ) metastatic lesions, even in the absence of symptoms (4). Weichselbaum and Hellman (4) theorized that before development of widespread metastases, malignant cells harbor "restricted metastatic potential" and occupy only a limited number of sites apart from the primary tumor. Theoretically, aggressive local therapy during this time could delay the need for systemic therapies and their attendant toxicities and/or prolong progression-free survival.

Stereotactic body radiation therapy (SBRT) is a sophisticated form of external beam radiation therapy that delivers ablative doses of radiation with high precision while minimizing collateral damage to surrounding normal tissues (5). Stereotactic body radiation therapy is a potentially useful radiotherapeutic modality in the pursuit of durable control in the setting of metastatic disease because it is convenient, noninvasive, and typically does not delay the administration of systemic therapies (6-9). Our initial institutional results were previously reported for oligometastatic PCa ( 5 PCa lesions on diagnostic imaging) treated with SBRT (6). Herein, we report our updated institutional outcomes with SBRT for oligometastatic $\mathrm{PCa}$, and we additionally explore patient and treatment factors that are associated with SBRT outcomes.

\section{Methods and Materials}

With the approval of our institutional review board, we queried a prospectively collected database of patients treated with SBRT from April 1, 2009 through January 31, 2014. All patients in the database had provided written, informed consent allowing their medical records to be used in research studies. Inclusion criteria were as follows: biopsy-proven PCa, diagnostic imaging consistent with metastases, and at least 3 months of follow-up with postSBRT PSA tests and imaging. The majority of patients had $\mathbf{5}$ sites of disease; 2 patients with more than 10 lesions were re-treated with SBRT for palliation of lesions that had not 
responded to conventionally fractionated radiation therapy and were also included in the analysis.

Patients at our institution are generally offered SBRT if they have an Eastern Cooperative Oncology Group performance status (10) of 0-1, limited systemic options, and $<5$ metastases at the time of consultation, all of which measure $<5 \mathrm{~cm}$ in greatest axial dimension and can be appropriately targeted with SBRT. Preference is given to patients who have progressed despite systemic therapy, although patients who refuse or cannot tolerate systemic therapy may still be offered SBRT. Metastases that required emergent therapy are not candidates for SBRT.

\section{SBRT technique}

Patients were immobilized using the Body-Fix whole-body or thoracic-T double-vacuum immobilization system (Medical Intelligence Medizintechnik, Schwabmünchen, Germany) and simulated using a Light Speed RT 16-slice computed tomography (CT) scanner (GE Health Care, Chicago, IL). Magnetic resonance imaging (MRI) or ${ }^{11} \mathrm{C}$-choline positron emission tomography (PET)/CT images were fused with the planning CT to assist in delineation of the gross tumor volume (GTV) and organs at risk, as necessary. Gross tumor volume was defined as the sum of the abnormalities noted on PET, MRI T1 post-gadolinium sequences, and/or CT. For spine lesions, the clinical tumor volume (CTV) included the GTV, the remainder of the involved anatomic section, and the adjacent anatomic sections of the vertebral body, similar to, and subsequently in accordance with, the International Spine Radiosurgery Consortium (11). For nonspinal osseous lesions, the CTV was a 1-cm expansion from the GTV within contiguous bone, in the absence of tumor extension into soft tissue. For nodal lesions and soft-tissue extension, the CTV was typically a 5-mm expansion from the GTV, with appropriate anatomic modifications. Most commonly, 2 planning target volumes (PTVs) were delineated, with GTV alone or with a small $(<2-\mathrm{mm})$ margin treated to the prescription (high) dose and a second, low-dose PTV, typically defined as the abovedescribed CTV plus $2 \mathrm{~mm}$. For the purpose of analysis, treatment prescription was reported as the high-dose PTV prescription.

A dose of 16 Gy in 1 fraction was initially used, based on the calculation of a biologically effective dose equivalent to $80 \mathrm{~Gy}$ delivered in 2-Gy fractions (calculated with $\alpha / \beta$ of 1.5 ). Single-fraction therapy was chosen for several reasons: $(1)$ it is more convenient for patients; (2) it is less expensive for payers; and (3) the proposed $\alpha / \beta$ of 1.5 for prostate cancer favors larger fraction size. The prescription dose was later increased on the basis of witnessed progression in some metastases that were treated to $16 \mathrm{~Gy}$, and given experience in treating other histologies to higher doses.

Treatment planning was performed using Eclipse treatment planning software (Varian Medical Systems, Palo Alto, CA). Stereotactic body radiation therapy was delivered with 3dimensional, conformal, static intensity modulated radiation therapy or volumetric modulated arc therapy, according to location and proximity to critical structures. Dose constraints for organs at risk were similar to, and subsequently in accordance with, the American Association of Physicists in Medicine Task Group 101 constraints (12). Daily image guidance was used for all treatments, and images were reviewed by the treating 
physician before treatment delivery. At the discretion of the treating radiation oncologist, image guidance was performed with ExacTRAC (Novalis) Westchester, IL or cone-beam CT.

\section{Follow-up}

Patients had serial follow-up at 3-month intervals, with PSA measured at least as often. Repeat imaging with ${ }^{11} \mathrm{C}$-choline PET/CT, MRI, or bone scan was conducted at 3- to 6month intervals after SBRT. The choice of imaging for follow-up was at the discretion of the physicians managing the systemic therapy options for the patient. Most commonly, the same imaging that initially detected the metastasis(es) was repeated in follow-up. In this report, metastasis control (MC) is reported only when imaging of the treated lesion was available.

\section{Statistical analysis}

Clinical endpoints reported are MC, biochemical progression-free survival (BPFS), distant progression-free survival (DPFS), and overall survival (OS). We defined MC as the lack of tumor progression within the high- or low-dose PTV on follow-up imaging. Marginal failures that occurred outside of the PTV were considered controlled for the purpose of our $\mathrm{MC}$ analysis, to allow meaningful analysis of the association between prescribed dose and control. However, marginal failures are described in detail. Biochemical failure was defined as (I) in the case of the initial decline from baseline after SBRT, the first PSA increase that was $25 \%$ and $\geq 2 \mathrm{ng} / \mathrm{mL}$ above the nadir, or an increase that was $225 \%$ and greater than the pretreatment PSA value, as confirmed by a second value 3 or more weeks later; or (2) in the case of no initial decline from baseline, a PSA increase that was $\geq 25 \%$ and $\geq 2 \mathrm{ng} / \mathrm{mL}$ greater than baseline after 3 months if baseline PSA was $\geq 2 \mathrm{ng} / \mathrm{mL}$, or PSA increase that was $\geq 25 \%$ after 3 months if baseline PSA was $<2 \mathrm{ng} / \mathrm{mL}$ (13). Distant progression-free survival was defined as no progression of untreated metastases and no new metastases on follow-up imaging. Nonlocalized disease was defined as N1 or M1. Stereotactic body radiation therapy-related toxicities were scored using the Common Terminology Criteria for Adverse Events v4.0 (14). Late toxicity was defined as occurring more than 3 months after SBRT.

The Kaplan-Meier method was used to estimate the rates of MC, BPFS, DPFS, and OS. Outcomes were reported at 2 years and were calculated from the date of SBRT. To assess the relationship between patient and treatment variables and outcomes, the log-rank test and the Cox proportional hazards test were used. Nominal and dichotomized continuous variables were assessed for significance using log-rank analysis. Dichotomized continuous variables were separated at the median value, unless otherwise specified. Continuous variables were analyzed with the Cox proportional hazards test. Analysis of MC was performed with each treated lesion considered individually $(\mathrm{n}=81)$, whereas BPFS, DPFS, and OS were analyzed with each individual being considered only once, using the earliest SBRT date as the benchmark treatment. Variables assessed for impact on outcomes were age, Gleason score, PSA level at diagnosis and treatment, $\mathrm{T}$ stage, $\mathrm{N}$ stage, $\mathrm{M}$ stage, localized versus nonlocalized disease at diagnosis, receipt of androgen deprivation therapy, number of metastases at the time of SBRT, date of SBRT, and GTV volume. Variables that were significant were then analyzed using multivariate Cox proportional hazards analysis. 
Associations between variables and outcomes were considered significant if $P$ values were $<.05$. Statistical analysis was performed using JMP 9.0 (SAS Institute, Cary, NC).

\section{Results}

Patients

Sixty-nine patients with 87 metastatic PCa lesions were initially identified. Three men with 6 treated lesions were excluded because of inadequate follow-up. Thus, 66 patients with 81 metastatic PCa lesions were included in this analysis. Median duration of follow-up was 16 months (range, 3.3-49.2 months). Patients were castrate resistant at the time of treatment for 50 lesions $(62 \%)$. Additional patient and disease characteristics at diagnosis and the time of SBRT are shown in Tables 1 and 2, respectively. Imaging modalities used to detect metastatic disease included ${ }^{11} \mathrm{C}$-choline PET/CT $(\mathrm{n}=46$ [70\%]), MRI $(\mathrm{n}=8$ [12\%]), CT $(\mathrm{n}=2$ [3\%]), and bone scan $(\mathrm{n}=10[15 \%])$.

Stereotactic body radiation therapy was delivered in a single fraction for 71 lesions (88\%). The median dose for single-fraction regimens was $16 \mathrm{~Gy}$ (range, 16-24 Gy). The remaining lesions were treated with $30 \mathrm{~Gy}$ in 3 fractions (6 lesions [7\%]) or $50 \mathrm{~Gy}$ in 5 fractions (4 lesions [5\%]). All lesions treated with $50 \mathrm{~Gy}$ in 5 fractions were soft-tissue targets (lymph nodes [ $\mathrm{n}=3$ ]; liver [ $\mathrm{n}=1]$ ). All lesions treated with $30 \mathrm{~Gy}$ in 3 fractions had received prior radiation. No other fractionation pattern was used for retreatment. The specific SBRT fractionation patterns used are shown in Table 3.

\section{Survival}

At 2 years, estimated MC rate by lesion was $82 \%$. Two-year BPFS, DPFS, and OS rates by patient were $54 \%, 45 \%$, and $83 \%$, respectively.

\section{Metastasis control}

Factors that were significantly associated with $\mathrm{MC}$ were $\mathrm{M}$ stage at the time of initial diagnosis (M0 vs M1; $P \leq 001$ ), risk group at the time of initial diagnosis (localized vs nonlocalized disease; $P=.02$ ), and dichotomized treatment dose (16 vs $\geq 18 \mathrm{~Gy} ; P \leq 001)$. No lesion being retreated progressed after SBRT. On multivariate analyses, only the dichotomized treatment dose ( $16 \mathrm{vs} \geq 18 \mathrm{~Gy}$ ) remained significant, with lesions treated with $\geq 18$ Gy showing superior MC. At 2 years, Kaplan-Meier estimated MC for lesions treated with $16 \mathrm{~Gy}$ was $58 \%$; for those treated with $\geq 18 \mathrm{~Gy}$, the $\mathrm{MC}$ rate was $95 \%$. Lesions treated with $18 \mathrm{~Gy}$ in a single fraction had $88 \% \mathrm{MC}$ at 2 years. When the population was dichotomized into lesions treated with $\geq 18$ Gy versus $>18 \mathrm{~Gy}$, the association between lower dose and increased risk of local failure persisted, with estimated risk at 2 years of $69 \%$ and $100 \%$, respectively (hazard ratio, 5.6; $P=.18$ ). No lesion treated with $30 \mathrm{~Gy}$ in 3 fractions or with 50 Gy in 5 fractions progressed.

Two of 21 lesions treated with 18 Gy in 1 fraction recurred within the treated volume, the first at 22 months and the second at 44 months. All relapses occurred within the high-dose PTV, except for 1 patient with a sternal lesion that was treated with $16 \mathrm{~Gy}$ in 1 fraction. The high-dose PTV had received 16 Gy and a low-dose PTV received 12 Gy, and the lesion 
progressed within the low-dose (12 Gy) PTV isodose. This lesion was categorized as having progressed for our final analysis; however, if excluded from analysis, the association between dichotomized dose and MC remained significant.

As mentioned above, 3 marginal failures occurred, with tumor recurrence just outside of the PTV. The first was a lesion at the L4 vertebral body, originally treated with a 24-Gy dose in 1 fraction; the tumor recurred in the left lamina, between the 10- and 16-Gy isodose lines. The second was an ischial lesion initially treated with $16 \mathrm{~Gy}$ in 1 fraction that had recurrence at 2 locations: 1 between the 7.5- and 10-Gy isodose regions and the second in the 2.5-Gy isodose region. Finally, a pelvic lesion treated with $16 \mathrm{~Gy}$ in 1 fraction had recurrence within the 12-Gy isodose region. We considered these lesions to be locally controlled for the purpose of our analysis, to allow sound determination of dose-control relationships. When considered as local failures, the estimated 2-year MC was 76\%. The significance of our analyses remained unchanged.

\section{Other outcomes}

Prostate-specific antigen at the time of SBRT was associated with BPFS $(P \leq 001)$, with higher pretreatment values predicting a greater chance of posttreatment biochemical progression. No other variable was significantly associated with BPFS. Distant progressionfree survival was associated with PSA at the time of SBRT $(P \leq 001)$, total number of metastases at the time of SBRT $(P \leq 001)$, and location of treated metastases (bone vs lymph nodes, $P<.001)$. On multivariate analysis, PSA at the time of SBRT and the total number of metastases remained significant, with higher pre-SBRT PSA value and greater number of metastatic lesions at the time of SBRT being associated with lower rates of distant control $(P \leq 05)$. The total number of metastases at the time of SBRT was associated with OS, with more metastases associated with poorer OS $(P \leq 001)$.

\section{Toxicity}

Eight patients (12\%) had acute pain flares, $6(9 \%)$ with grade 1 pain flare and $2(3 \%)$ with grade 2 pain flare. No additional acute toxicities were reported. No grade 2 or greater late toxicities were reported.

\section{Discussion}

This series serves as an update to our institution's previously published data regarding the efficacy of SBRT for metastatic PCa (6). The previously reported MC rate was $100 \%$, with a median follow-up of 6 months for 17 patients with 21 lesions. With longer follow-up and more patients treated, we observed 9 local failures in 8 patients, with an estimated MC rate of $82 \% 2$ years after SBRT. Multivariate testing showed that SBRT dose (16 Gy vs $\geq 18 \mathrm{~Gy}$ ) was the only patient or treatment characteristic associated with MC, with patients treated with $\geq 18$ Gy having superior MC. No local recurrence was seen with a total dose $>18 \mathrm{~Gy}$. This is a critical finding, suggesting that when single-fraction SBRT regimens are used for metastatic PCa, doses $\geq 18$ Gy should be considered, to achieve durable MC. All dose levels were well tolerated in this series, with no grade 2 or greater late toxicities reported to date. 
Investigators have reported outcomes of various histologies treated with SBRT, including traditionally radioresistant tumors, such as renal cell carcinoma. Similarly, a wide range of metastatic sites has been treated, including osseous, pulmonary, hepatic, nodal, and softtissue sites. Metastasis control may be affected by tumor and treatment characteristics, making cross-comparison between reports of different primary disease types and treatment regimens challenging; however, a dose-response pattern has emerged in the SBRT literature for treatment of various primary tumors $(15,16)$ and metastatic disease $(16-18)$. Salama et al (17) performed a prospective dose-escalation study in patients receiving SBRT for oligometastatic malignancy, with patients treated with 24 to $48 \mathrm{~Gy}$ in 3 fractions. Patients treated with $24 \mathrm{~Gy}$ had an MC rate of $46 \%$, whereas rates for patients treated with higher doses ranged from $62.5 \%$ to $100 \%$ (17). Greco et al (18) reported outcomes of 126 metastases in 103 patients treated with single-fraction SBRT; they described an association between local relapse-free survival (LRFS) and prescription dose, with patients receiving 23 to 24 Gy having an LRFS rate of $82 \%$, whereas patients treated with a low dose (18-20 Gy) had an LRFS rate of $25 \%$. Importantly, much of this difference was driven by patients with renal cell carcinoma, who had very poor LRFS with low-dose SBRT (18-20 Gy).

The variability of the relationship between dose-response and histology, as illustrated by Greco et al (18), underscores the importance of analyzing data in a histology-specific manner when determining the most appropriate prescription dose for a particular type of tumor. To our knowledge, before the present study, no clear dose-response data existed for oligometastatic PCa. Jereczek-Fossa et al (19) recently reported outcomes of a cohort of patients treated for recurrent or metastatic PCa using a range of SBRT doses, with a median dose of $30 \mathrm{~Gy}$ in 4.5 fractions. The 30-month progression-free survival rate was $42.6 \%$, and no clear dose-response data emerged. Muacevic et al (20) reported 95.5\% 2-year control of osseous PCa metastasis treated with single-fraction SBRT, with doses ranging from 16.5 to $22 \mathrm{~Gy}$. Reports from other groups have indicated $100 \% \mathrm{MC}$ with schedules of $50 \mathrm{~Gy}$ in 10 fractions $(21,22)$ and $30 \mathrm{~Gy}$ in 3 fractions $(22,23)$.

Our data show that higher pre-SBRT PSA levels were associated with a greater risk of biochemical failure $(P \leq 001)$ and distant progression $(P \leq 001)$. Furthermore, the more metastases a patient had at the time of SBRT, the more likely they would have distant progression $(P \leq 001)$. Men with higher PSA values and more metastatic lesions at the time of SBRT are more likely to harbor subclinical disease that becomes evident during followup. Because of the impact of hormonal and systemic therapy on BPFS, DPFS, and OS and the various systemic agents used in this population, no conclusions can be drawn regarding the impact of SBRT on BPFS, DPFS, and OS.

The present study was limited by its retrospective nature, by the relatively short duration of follow-up, and by the inability to draw conclusions regarding the impact of SBRT on survival outcomes. Analysis of DPFS, BPFS, and OS were limited by the heterogeneity of systemic therapies used. A prior report aimed to prove that SBRT may be used to defer initiation of hormonal therapy (21). We were unable to perform such an analysis because most of our patients were undergoing concurrent androgen deprivation therapy at the time of SBRT. Despite these limitations, this study is a valuable contribution to the literature because it reports outcomes for a large cohort of patients with oligometastatic PCa. Analysis 
of this histologically homogeneous group allowed us to define a dose-response relationship in which patients treated with 16 Gy were more likely to have local failure than those treated with $\geq 18$ Gy.

\section{Conclusion}

Stereotactic body radiation therapy is a feasible treatment modality for oligometastatic PCa. In this series, patients treated with $\geq 18$ Gy had improved MC compared with the cohort treated with $16 \mathrm{~Gy}$. Thus, if single-fraction treatment is recommended, we suggest using single-fraction doses $\geq 18 \mathrm{~Gy}$ when feasible. Metastasis control seems to be superior with doses >18 Gy (relative to $18 \mathrm{~Gy}$ ); however, the incremental gain in MC must be weighed against possible increased risk of toxicity. Few marginal failures were seen in this cohort, suggesting that our treatment volume delineation was appropriate. Investigation into the relationship between SBRT and systemic therapies is warranted, especially in patients with castration-refractory PCa. Furthermore, evaluation of the efficacy of metastasis-directed therapy in improving clinical outcomes including survival and occurrence of skeletal events is critical and ongoing (24).

\section{References}

1. Siegel R, Ma J, Zou Z, et al. Cancer statistics, 2014 [erratum in: CA Cancer J Clin 2014;64:364]. CA Cancer J Clin. 2014; 64:9-29. [PubMed: 24399786]

2. Fizazi K, Scher HI, Molina A, et al. Abiraterone acetate for treatment of metastatic castrationresistant prostate cancer: Final overall survival analysis of the COI-AA-301 randomized, doubleblind, placebo-controlled phase 3 study. Lancet Oncol. 2012; 13:983-992. [PubMed: 22995653]

3. Scher HI, Fizazi K, Saad F, et al. Increased survival with enzalutamide in prostate cancer after chemotherapy. N Engl J Med. 2012; 367:1187-1197. [PubMed: 22894553]

4. Weichselbaum RR, Hellman S. Oligometastases revisited. Nat Rev Clin Oncol. 2011; 8:378-382. [PubMed: 21423255]

5. Chang BK, Timmerman RD. Stereotactic body radiation therapy: A comprehensive review. Am J Clin Oncol. 2007; 30:637-644. [PubMed: 18091059]

6. Ahmed KA, Barney BM, Davis BJ, et al. Stereotactic body radiotherapy in the treatment of oligometastatic prostate cancer. Front Oncol. 2013; 2:2015.

7. Lo SS, Fakiris AJ, Chang EL, et al. Stereotactic body radiation therapy: A novel treatment modality [erratum in: Nat Rev Clin Oncol 2010;7:422; dosage error in article text]. Nat Rev Clin Oncol. 2010; 7:44-54. [PubMed: 19997074]

8. Gerszten PC, Burton SA, Ozhasoglu C, et al. Radiosurgery for spinal metastases: Clinical experience in 500 cases from a single institution. Spine (Phila Pa 1976). 2007; 32:193-199. [PubMed: 17224814]

9. Rusthoven KE, Kavanagh BD, Cardenes H, et al. Multi-institutional phase I/II trial of stereotactic body radiation therapy for liver metastases. J Clin Oncol. 2009; 27:1572-1578. [PubMed: 19255321]

10. Oken MM, Creech RH, Tormey DC, et al. Toxicity and response criteria of the Eastern Cooperative Oncology Group. Am J Clin Oncol. 1982; 5:649-655. [PubMed: 7165009]

11. Cox BW, Spratt DE, Lovelock M, et al. International Spine Radiosurgery Consortium consensus guidelines for target volume definition in spinal stereotactic radiosurgery. Int J Radiat Oncol Biol Phys. 2012; 83:e597-e605. [PubMed: 22608954]

12. Benedict SH, Yenice KM, Followill D, et al. Stereotactic body radiation therapy: The report of AAPM Task Group 101 [erratum in: Med Phys 2012;39:563; dosage error in article text]. Med Phys. 2010; 37:4078-4101. [PubMed: 20879569] 
13. Scher HI, Halabi S, Tannock I, et al. Design and end points of clinical trials for patients with progressive prostate cancer and castrate levels of testosterone: Recommendations of the Prostate Cancer Clinical Trials Working Group. J Clin Oncol. 2008; 26:1148-1159. [PubMed: 18309951]

14. US Department of Health and Human Services, National Institutes of Health, National Cancer Institute. Common Terminology Criteria for Adverse Events (CTCAE), version 4.0. Available at: http://evs.nci.nih.gov/ftp1/CTCAE/CTCAE_4.03_2010-06-14_QuickReference_5x7.pdf. Accessed May 19, 2015

15. Hof H, Muenter M, Oetzel D, et al. Stereotactic single-dose radiotherapy (radiosurgery) of early stage nonsmall-cell lung cancer (NSCLC). Cancer. 2007; 110:148-155. [PubMed: 17516437]

16. McCammon R, Schefter TE, Gaspar LE, et al. Observation of a dose-control relationship for lung and liver tumors after stereotactic body radiation therapy. Int J Radiat Oncol Biol Phys. 2009; 73:112-118. [PubMed: 18786780]

17. Salama JK, Hasselle MD, Chmura SJ, et al. Stereotactic body radiotherapy for multisite extracranial oligometastases: Final report of a dose escalation trial in patients with 1 to 5 sites of metastatic disease. Cancer. 2012; 118:2962-2970. [PubMed: 22020702]

18. Greco C, Zelefsky MJ, Lovelock M, et al. Predictors of local control after single-dose stereotactic image-guided intensity-modulated radiotherapy for extracranial metastases. Int J Radiat Oncol Biol Phys. 2011; 79:1151-1157. [PubMed: 20510537]

19. Jereczek-Fossa BA, Beltramo G, Fariselli L, et al. Robotic image-guided stereotactic radiotherapy, for isolated recurrent primary, lymph node or metastatic prostate cancer. Int J Radiat Oncol Biol Phys. 2012; 82:889-897. [PubMed: 21277113]

20. Muacevic A, Kufeld M, Rist C, et al. Safety and feasibility of image-guided robotic radiosurgery for patients with limited bone metastases of prostate cancer. Urol Oncol. 2013; 31:455-460. [PubMed: 21481619]

21. Berkovic P, De Meerleer G, Delrue L, et al. Salvage stereotactic body radiotherapy for patients with limited prostate cancer metastases: Deferring androgen deprivation therapy. Clin Genitourin Cancer. 2013; 11:27-32. [PubMed: 23010414]

22. Decaestecker K, De Meerleer G, Lambert B, et al. Repeated stereotactic body radiotherapy for oligometastatic prostate cancer recurrence. Radiat Oncol. 2014; 9:135. [PubMed: 24920079]

23. Casamassima F, Masi L, Menichelli C, et al. Efficacy of eradicative radiotherapy for limited nodal metastases detected with cholic PET scan in prostate cancer patients. Tumori. 2011; 97:49-55. [PubMed: 21528664]

24. Decaestecker K, De Meerleer G, Ameye F, et al. Surveillance or metastasis-directed therapy for oligometastatic prostate cancer recurrence (STOMP): Study protocol for a randomized phase II trial. BMC Cancer. 2014; 14:671. [PubMed: 25223986] 


\section{Summary}

Use of stereotactic body radiation therapy (SBRT) has been reported in the treatment of oligometastatic prostate cancer; however, the optimal dose fractionation has not been elucidated. This analysis indicates that single-fraction SBRT using $16 \mathrm{~Gy}$ is associated with poorer local control relative to single-fraction SBRT using $\geq 18 \mathrm{~Gy}$ or higher-dose, multifraction SBRT regimens. Toxicity was acceptable in this series. 


\section{Table 1}

Patient and disease characteristics at diagnosis $(n=66)$

\begin{tabular}{|c|c|}
\hline Characteristic & Value \\
\hline Age at diagnosis (y), median (range) & $61.4(44-78)$ \\
\hline \multicolumn{2}{|l|}{ Gleason score, $\mathrm{n}(\%)$} \\
\hline 6 & $3(4.6)$ \\
\hline 7 & $30(45.5)$ \\
\hline 8 & $9(13.6)$ \\
\hline 9 & $21(31.8)$ \\
\hline 10 & $2(3)$ \\
\hline Unknown & $1(1.5)$ \\
\hline \multicolumn{2}{|l|}{ PSA } \\
\hline Initial value (ng/mL), median (range) & $8.4(1.9-193)$ \\
\hline \multicolumn{2}{|l|}{ PSA value $(\mathrm{ng} / \mathrm{mL}), \mathrm{n}(\%)$} \\
\hline $0-5$ & $15(22.7)$ \\
\hline$>5-10$ & $14(21.2)$ \\
\hline$>10-20$ & $11(16.7)$ \\
\hline$>20$ & $12(18.2)$ \\
\hline Unknown & $14(21.2)$ \\
\hline \multicolumn{2}{|l|}{ pT stage, n (\%) } \\
\hline pT2 & $25(37.9)$ \\
\hline pT3 & $32(48.5)$ \\
\hline pT4 & $2(3)$ \\
\hline Unknown & $7(10.6)$ \\
\hline \multicolumn{2}{|l|}{$\mathrm{N}$ stage, $\mathrm{n}(\%)$} \\
\hline No & $53(80.3)$ \\
\hline $\mathrm{N} 1$ & $13(19.7)$ \\
\hline \multicolumn{2}{|l|}{ M stage, n (\%) } \\
\hline M0 & $58(87.9)$ \\
\hline M1 & $6(9.1)$ \\
\hline Unknown & $2(3)$ \\
\hline \multicolumn{2}{|l|}{ D'Amico risk category, n (\%) } \\
\hline Low risk & $1(1.5)$ \\
\hline Intermediate risk & $12(18.2)$ \\
\hline High risk & $31(47)$ \\
\hline Node positive & $9(13.6)$ \\
\hline Metastatic & $6(9.1)$ \\
\hline Unknown & $7(10.6)$ \\
\hline \multicolumn{2}{|l|}{ Dichotomized risk group, n (\%) } \\
\hline Localized & 44 (66.7) \\
\hline Nonlocalized $^{*}$ & $15(22.7)$ \\
\hline Unknown & $7(10.6)$ \\
\hline
\end{tabular}




\begin{tabular}{lc}
\hline Characteristic & Value \\
\hline Primary treatment & \\
RP alone & $20(30.3)$ \\
RT alone & $3(4.5)$ \\
RP and adjuvant RT & $9(13.6)$ \\
RP and salvage RT & $26(39.4)$ \\
RT and salvage RP & $1(1.5)$ \\
RT and cryotherapy & $1(1.5)$ \\
Medical (chemotherapy) & $6(9.1)$ \\
Adjuvant androgen-deprivation therapy, n (\%) & $42(63.6)$ \\
\hline
\end{tabular}

Abbreviations: PSA = prostate-specific antigen; $\mathrm{RP}=$ radical prostatectomy; $\mathrm{RT}=$ radiation therapy .

N1- and/or M1-stage disease. 


\section{Table 2}

Patient and disease characteristics at the time of stereotactic body radiation therapy

\begin{tabular}{|c|c|c|}
\hline Characteristic & Patient $(n=66)$ & Lesion $(\mathrm{n}=\mathbf{8 1})$ \\
\hline Overall PSA (ng/mL), median (range) & $1.9(0-32)$ & $1.9(0-32)$ \\
\hline \multicolumn{3}{|l|}{ PSA $(\mathrm{ng} / \mathrm{mL}), \mathrm{n}(\%)$} \\
\hline $0-5$ & $51(77.3)$ & $60(74.1)$ \\
\hline$>5-10$ & $9(13.6)$ & $12(14.8)$ \\
\hline$>10-20$ & $5(7.6)$ & $8(9.9)$ \\
\hline$>20$ & $1(1.5)$ & $1(1.2)$ \\
\hline \multicolumn{3}{|l|}{ Total no. of metastases, no. of patients (\%) } \\
\hline 1 & $46(69.7)$ & $52(64.2)$ \\
\hline 2 & $12(18.2)$ & $18(22.2)$ \\
\hline 3 & $3(4.5)$ & $4(4.9)$ \\
\hline 4 & $1(1.5)$ & $1(1.2)$ \\
\hline 5 & $2(3.0)$ & $4(4.9)$ \\
\hline $6-10$ & $0(0)$ & $0(0)$ \\
\hline$>10$ & $2(3.0)$ & $2(2.5)$ \\
\hline \multicolumn{3}{|l|}{ Regions treated, n (\%) } \\
\hline \multicolumn{3}{|l|}{ Bone } \\
\hline Thoracic spine & $7(10.6)$ & $9(11.1)$ \\
\hline Lumbar spine & $9(13.6)$ & $10(12.3)$ \\
\hline Sacrum & $13(19.7)$ & $16(19.8)$ \\
\hline Pelvis & $21(31.8)$ & $24(29.6)$ \\
\hline Sternum & $3(4.5)$ & $4(4.9)$ \\
\hline Rib & $5(7.6)$ & $8(9.9)$ \\
\hline Acromion & $2(3.0)$ & $2(2.5)$ \\
\hline Scapula & $1(1.5)$ & $1(1.2)$ \\
\hline Lymph node & $5(7.6)$ & $6(7.4)$ \\
\hline Liver & $1(1.5)$ & $1(1.2)$ \\
\hline \multicolumn{3}{|l|}{ Primary indication, n (\%) } \\
\hline Definitive & $57(86.4)$ & $69(85.2)$ \\
\hline Palliative & $9(13.6)$ & $12(14.8)$ \\
\hline
\end{tabular}

Abbreviation as in Table 1. 
Table 3

Specific stereotactic body radiation therapy fractionation patterns by lesion

\begin{tabular}{lcc}
\hline Specific PTV dose $(\mathbf{G y}) / \mathbf{n o .}$ of fractions $^{*}$ & Lesions treated, $\mathbf{n}(\boldsymbol{\%})(\mathbf{n}=\mathbf{8 1})$ & $\mathbf{G T V}\left(\mathbf{c m}^{\mathbf{3}}\right)$, mean $(\mathbf{r a n g e})$ \\
\hline $16 / 1$ & $38(46.9)$ & $10.1(0.8-87.3)$ \\
$18 / 1$ & $21(25.9)$ & $15.4(0.6-101.9)$ \\
$19 / 1$ & $1(1.2)$ & $25.5(\ldots)$ \\
$24 / 1$ & $11(13.6)$ & $14.6(0.3-71.2)$ \\
$30 / 3$ & $6(7.4)$ & $13.5(3.0-41.6)$ \\
$50 / 5$ & $4(4.9)$ & $28.1(3.1-86.0)$ \\
\hline
\end{tabular}

Abbreviations: $\mathrm{GTV}=$ gross tumor volume; PTV = planning target volume .

Eighteen patients were treated with $16 \mathrm{~Gy} ; 43$ patients received $\geq 18 \mathrm{~Gy}$. 